\title{
HOLE BURNING ON AN IONIC DYE IN A LANGMUIR-BLODGETT MONOLAYER
}

\author{
Michel ORRIT, Jacky BERNARD \\ Centre de Physique Moléculaire Optique et Hertzienne CNRS et Université de Bordeaux, 33405 Talence, France \\ and \\ Dietmar MÖBIUS \\ Max-Planck-Institut für Biophysikalische Chemie, Am Fassberg, D-3400 Göttingen, Federal Republic of Germany
}

Received 5 December 1988; in final form 20 January 1989

\begin{abstract}
We report on the observation of persistent holes in the excitation spectrum of a single monolayer, using the ionic dye resorufin adsorbed on an ammonium salt monolayer. We observed a slow temperature dependence similar to that of 3D glasses. The holes can be used as sensitive surface probes to detect the tiny change in dielectric constant consecutive to immersion in superfluid helium.
\end{abstract}

\section{Introduction}

In recent years, a large number of potential applications for the optical properties of LangmuirBlodgett (LB) films have been put forward, ranging from non-linear devices to chemical sensors $[1,2]$ (for a review on optical properties of LB films, see ref. [3]). However, the structure and dynamics of these complex molecular systems are still ill known, and every new characterization method may be of interest. In this paper, we present our first results on high-resolution hole-burning spectroscopy performed on a single dye-containing LB film. We think this method could become a powerful tool for investigating $2 \mathrm{D}$ systems and interfaces.

The spectral hole burning of 3D dilute solutions has proved to be a sensitive method for studying matrix dynamics (related to matrix structure) as well as the effect of external perturbations [4,5]. Whereas a crystalline matrix shows a steep drop of the hole width at low temperatures, glasses exhibit weaker $T$ dependences such as $T^{n}$ with $1<n<2$ over wide temperature ranges below $20 \mathrm{~K}$ [6]. This specific behaviour of glasses is ascribed [7] to the so-called twolevel systems (TLS) which are low-energy (from 0 to a few $\mathrm{cm}^{-1}$ ) excitations specific to glasses. This raises the question of the behaviour in this respect of LB films which show a rather well-defined structure [8] (apparently better defined than that of glasses) while their conventional absorption and emission spectra look inhomogeneously broadened in much the same way as glass spectra [9]. Fluorescence line narrowing of perylene in LB-film assemblies has been reported by Bogner et al. [10].

Besides the interest of the holes for their own sake, sharp holes may be used as very sensitive probes of external perturbations such as electric $[11,12]$ or magnetic [13,14] fields, pressure [15] and mechanical stresses [16], etc. A second aim of the present work is to show that holes in monolayers can be used as sensitive surface probes.

\section{Experimental}

The samples were ordinary glass slides covered on both sides with a monolayer assembly consisting of a substrate layer of eicosylamine (EA), then the dyecontaining monolayer and a third covering layer of EA. We prepared and transferred all monolayers in Göttingen, according to experimental procedures described in earlier work from this laboratory [17]. The 
dye film was spread using a 1:50 mixture of the water-soluble sodium salt of resorufine (Reso) with dioctadecyldimethylammonium bromide (DOMA). This procedure first used with cyanine dyes [18] avoids working on a dye solution. We found that at least some of the soluble dye in its coloured form remained adsorbed on the monolayer after transfer.

The hole-burning apparatus in Bordeaux is of the fluorescence excitation type, similar to that used by Völker and coworkers [19] and will be described in more detail in a forthcoming publication. The excitation source is a single-mode ring dye laser ( $\mathrm{Co}-$ herent Radiation, model CR-699, maximum resolution $0.5 \mathrm{MHz}$ ) operated with rhodamine $6 \mathrm{G}$. The light intensity was regulated using a commercial electrooptic system (Conoptics, model Lass2). The sample, consisting of a stacking of 35 covered glass plates, was held in a U-shaped aluminum block about $3 \mathrm{~mm}$ thick. This block was placed in a helium-flow cryostat allowing temperatures down to $1.7 \mathrm{~K}$ by pumping on the liquid helium. At temperatures lower than $2.5 \mathrm{~K}$ the sample was immersed in superfluid helium, while it was swept by the helium gas flow above $2.5 \mathrm{~K}$. The glass plates were illuminated at Brewster's incidence. The weak emission was detected through a $630 \mathrm{~nm}$ red-pass Schott filter by a RCA C31034A photomultiplier tube operated in the photon-counting mode. Counts were acquired and scans accumulated by a multichannel analyzer (Tracor Northern, model TN-7200) and further processed on a microcomputer (Léanord, model Challenger).

\section{Results and discussion}

\subsection{Monolayer spectra}

Fig. 1 shows the absorption and emission spectra at room temperature of a monolayer assembly similar to those used in hole burning, but with a larger dye concentration (prepared from a Reso:DOMA 1:10 mixture). We checked the fluorescence excitation intensity of the dilute sample at helium temperature for a few discrete wavelengths and found no important difference from the absorption spectrum of fig. 1 . The dye surface density of the sample could be roughly estimated from the absorption $(0.02 \%$ for one plate) and an average distance between chromophores of about $100 \AA$ was deduced. This rather high surface density allowed a satisfactory detection of the emission and therefore of the holes, but was not small enough to rule out any energy transfer between dye molecules. From the oblique incidence spectra shown in fig. 1 , we inferred that the long axis of the dye anion lies in the monolayer plane, as is frequently the case [20]. We thus assumed that the dye anion is imbedded in the hydrophilic region between the DOMA and EA head groups, where its charge can be compensated by those of the ammonium ions.

\subsection{General conditions of hole burning and recording}

Holes could be burned in the inhomogeneously broadened band of resorufin in the monolayer matrix from about $606 \mathrm{~nm}$ to beyond $578 \mathrm{~nm}$, the deepest holes being obtained between 590 and $600 \mathrm{~nm}$. For the experiments reported here, we used a constant burning intensity of $10 \mu \mathrm{W} / \mathrm{cm}^{2}$, but we checked that no narrower hole could be obtained by burning with a lower intensity. Burning times ranged from a few seconds to a few minutes. The intensity used for recording was 100 times less than for burning. The measured hole depths varied from $1 \%$ to about $25 \%$, depending on burning energy and wavelength. On the red wing where the emission was very weak (beyond $600 \mathrm{~nm}$ ), the detectivity was impaired by emission from the glass plates.

Depth and width of the holes were determined by fitting a Lorentz curve to the experimental points, although the experimental peak consistently looked sharper around their maximum than a Lorentzian of equal width (see some hole shapes in fig. 4 below). We attribute this shape difference already seen on shallow holes to much slower burning at the hole maximum than on the wings. This effect would be due to dispersive kinetics of the burning process [21] and lead to a non-Lorentzian hole shape ${ }^{\# 1}$, of course blunter than the non-saturated Lorentzian hole, but sharper than a Lorentzian of equal width. This inter-

\footnotetext{
\#1 A simple model of hole saturation with a dispersive (logarithmic) burning kinetics can reproduce a hole shape sharper than Lorentzian.
} 


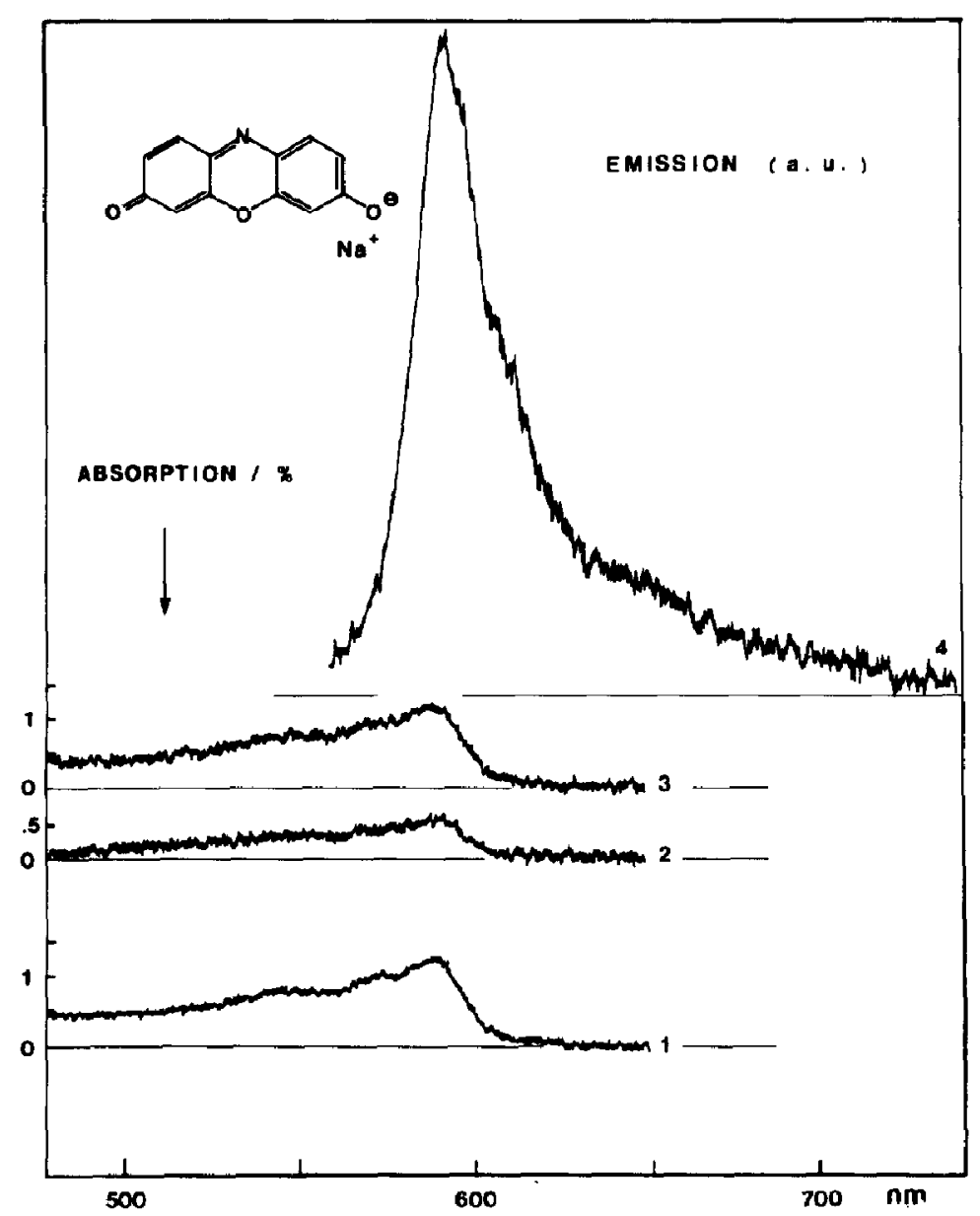

Fig. 1. Absorption (1-3) and emission (4) spectra of a LB film assembly at room temperature (see text) with the anionic dye resorufin adsorbed on an ammonium salt monolayer. (1) Normal incidence absorption; (2,3) absorption at $45^{\circ}$ incidence for the $p(2)$ and $s(3)$ polarizations. The ratio of spectra (2) and (3) shows that the long axis of resorufin lies in the film plane.

pretation is supported by a roughly linear dependence of the hole depth versus the logarithm of the irradiation time, also observed in resorufin in ethanol glass [22]. The holes rapidly broaden with burning time. Therefore, to measure the intrinsic hole width, we extrapolated to zero depth as shown in fig. 2. Due to noise, this gave rise to rather large error bars for the intrinsic width.

\subsection{Temperature dependence of the hole width}

The hole width was investigated between 1.7 and $10 \mathrm{~K}$ for the two wavelengths 592 and $600 \mathrm{~nm}$. The results are displayed in fig. 3. Despite the large error bars, we note that the temperature dependence is rather weak and reminds us more of glasses than of crystals.

Although measurements above $1.7 \mathrm{~K}$ do not determine the behavior at still lower temperatures (complex $T$ dependences were observed down to 0.05 K [23]), we tried to extrapolate the experimental points of fig. 3 to zero temperature and obtained the large extrapolated width of $2 \mathrm{GHz}$, independent of the wavelength within our accuracy. If this extrapolated width has a homogeneous origin, it is difficult to ascribe it to energy transfer to lower energy (ac- 


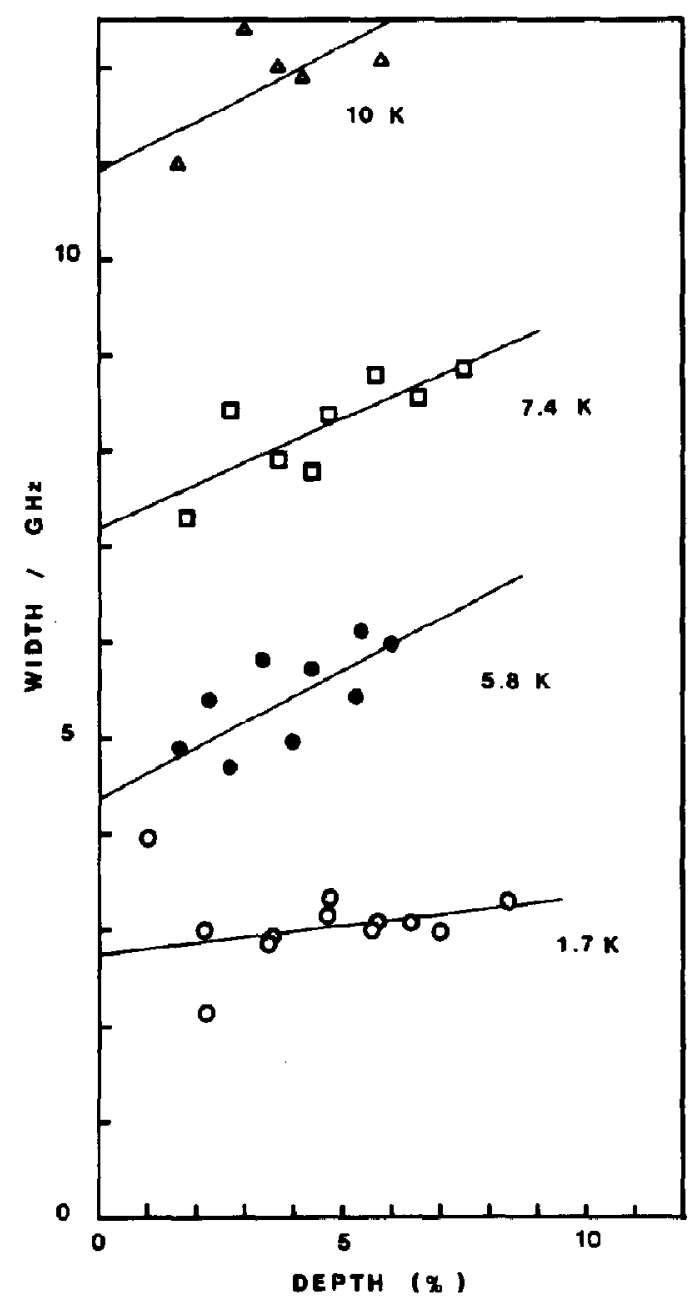

Fig. 2. Examples of width versus depth diagrams for holes burnt around $592 \mathrm{~nm}$ at different temperatures. Due to the scattered experimental points, the extrapolation to zero depth introduces a large inaccuracy in the width.

ceptor) resorufin ions (these should be much more numerous for the $592 \mathrm{~nm}$ than for the $600 \mathrm{~nm}$ sites). The width could be linked to other causes, e.g. to spectral diffusion during the time span between burning and recording [22].

By subtracting an extrapolated width of $2 \mathrm{GHz}$, a power law can be fitted to the $T$ dependence, i.c.

$\Gamma_{\text {hole }}=\Gamma_{\text {extr. }}+a T^{n}$

with $n=2.1 \pm 0.5$ at $592 \mathrm{~nm}$ and $n=1.6 \pm 0.5$ at 600 $\mathrm{nm}$. In spite of the large inaccuracy on these expo- nents, they seem larger than for $3 D$ glasses where $n=1.3$ is the rule. However, more precise measurements and more experience are needed to clear up the behavior of the width at low temperature in order to compare quantitatively the exponents of similar 2D and $3 \mathrm{D}$ systems.

\subsection{Immersion in liquid helium}

Since the molecules we are studying are separated from the cryostat atmosphere by only one EA monolayer (about $25 \AA$ thick), they should be affected by the composition of this atmosphere, in particular by the change from gaseous to liquid helium. We burned and recorded a deep hole at $2.6 \mathrm{~K}$ under helium gas atmosphere, then immersed the sample in superfluid liquid helium at $1.9 \mathrm{~K}$ and recorded the hole again, and finally heated up again and recorded the hole after complete emersion. All three hole spectra are shown in fig. 4.

First, we may note a reversible red-shift of the hole maximum when passing from gas to liquid. We measured this shift roughly by fitting Lorentz curves to the hole profiles, although the "immersed" hole shape looks asymmetric (this asymmetry is not easily seen in fig. 4). The value obtained for the shift averaged over six immersion experiments, $450-150 \mathrm{MHz}$, is in good agreement with the order of magnitude expected from a simple electric image model [24]. We calculated the energy shift of the dye transition, and found

$\Delta E=-\alpha \times 760 \mathrm{MHz}$,

where $\alpha$ is the unknown Debye-Waller factor of the zero-phonon line of resorufin in this matrix. We thus see that the spectral hole is sensitive to the slight change in dielectric permittivity (from 1.000 to 1.057) when passing from gas to liquid helium, even though the molecules are not in direct contact with the liquid.

The second important point is the apparent broadening of the hole after immersion, which seems at least partly irreversible, since the "emersed" hole is broader than the initial one. However, this last feature could be due to the heating necessary to remove the liquid, and we will discard it in the following. For the "immersed" hole in contrast, the cooling could only have caused a narrowing of the hole, while a 


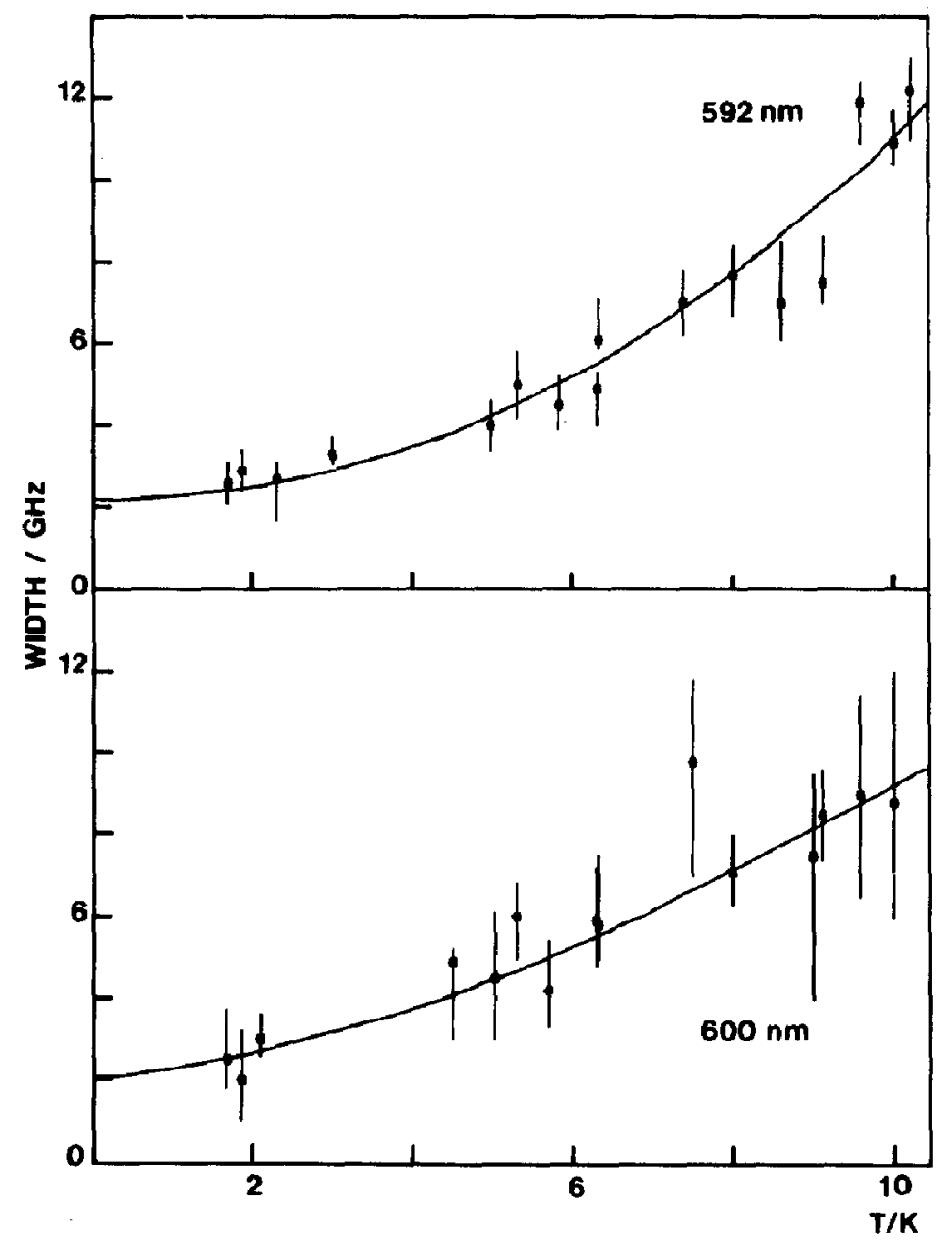

Fig. 3. Temperature dependence of the hole width for the two wavelengths 592 and $600 \mathrm{~nm}$. We note the residual width at low temperatures and the rather slow dependence, much evocative of 3D glassy systems. The solid lines are rough fits using the exponents given in the text.

broadening from 4.6 to $5.5 \mathrm{GHz}$ is observed. We again attribute this broadening to additional electrostatic images of matrix charges in the LB-film-liquid interface, this time acting by a static Stark effect on the dye anion. The Stark effect studies of Personov and coworkers [12] have shown the existence of strong internal fields around dye molecules, even in non-polar matrices. In our case, where the large charges of the dye counterions or of the ammonium ions of the matrix are thought to be randomly distributed in the hydrophilic region, random image fields react on the dye ions. Indeed, from the Stark coefficient of resorufin ( $\Delta \mu=0.2$ to 0.42 D) $[25,26]$, the shift due to the image field of a single counterion may be readily evaluated and is large enough to account for the observed broadening. According to the correlation of the direction of this field with the molecular axes, its effect could lead to additional shift and asymmetry of the hole. Thus, this effect should be taken into account for a quantitative calculation of the red-shift of the hole. Of course, much more work is needed to clear completely the different phenomena causing the observed shift, broadening and asymmetry of the "immersed" hole. 


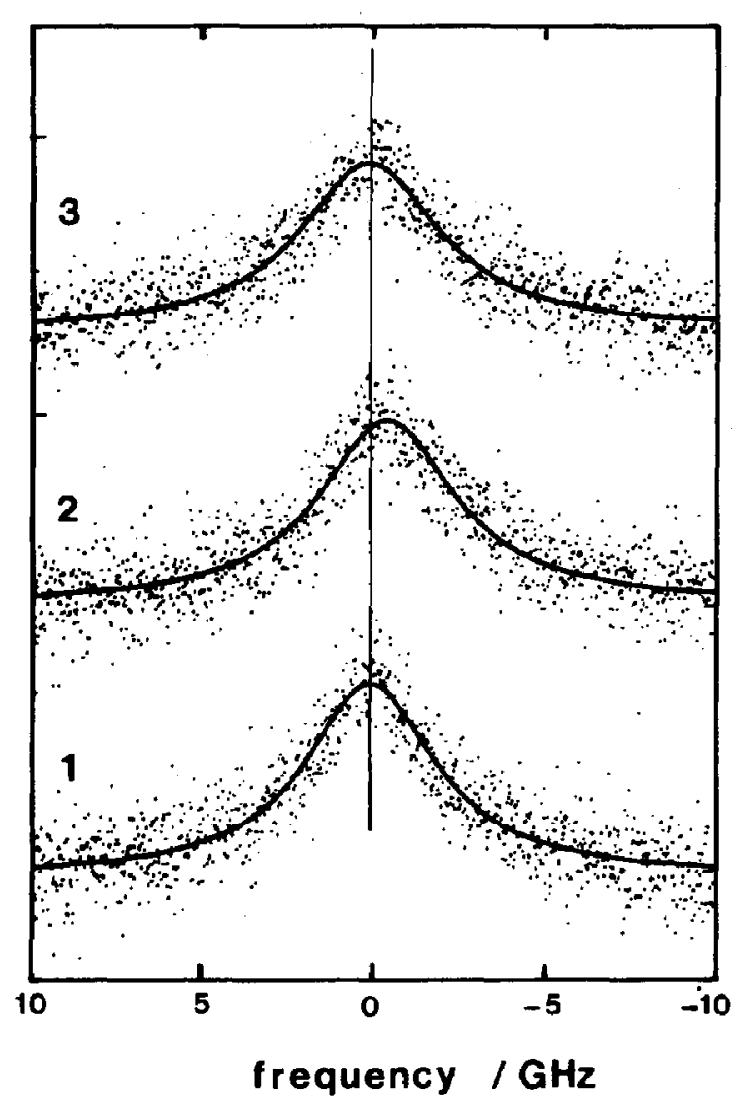

Fig. 4. Hole shapes in gaseous ( 1,3 ) and liquid (2) helium, including the experimental data (dots) and the adjusted Lorentzian (solid line). Spectrum (1) was recorded in the gas just after burning, (2) after immersion of the sample in superfluid helium, and (3) again in the gas after heating up and emersion of the sample. Note the reversible red-shift of the hole maximum and the broadening in the liquid. The apparent irreversibility of the broadening is possibly due to the thermal cycle.

\section{Acknowledgement}

This work was supported by the DRET (convention 87/259). We are grateful to Professor A. Ducasse, Dr. L. Sarger and Dr. E. Freysz for lcnding us the CR-699 and introducing us to its operation. We thank Dr. S. Völker, Dr. R. van den Berg and Dr. J.-P. Galaup for helpful information about the experimental hole-burning apparatus and the preparation of glassy samples and Professor R.I. Personov for stimulating discussions. Mrs. G. Debuch and Mr. $\mathrm{W}$. Zeiss prepared and characterized the monolayer samples. We thank them for their kind cooperation.

\section{References}

[1] D. Möbius, ed., Poceedings of the Third International Conference on Langmuir-Blodgett films, Göttingen, 1987, Thin Solid Films 159/160 (1988);

F. Kajzar, I.R. Girling and I.R. Peterson, Thin Solid Films 160 (1988) 209;

I. Ledoux, D. Josse, P. Fremaux, J.-P. Piel, G. Post, J. Zyss, T. MacLean, R.A. Hann, P.F. Gordon and S. Allen, Thin Solid Films 160 (1988) 217;

G. Marowsky, L.F. Chi, D. Möbius, R. Steinhoff, Y.R. Shen, D. Dorsch and B. Rieger, Chem. Phys. Letters 147 (1988) 420.

[2] J.H. Kim, T.M. Cotton and R.A. Uphaus, Thin Solid Films 160 (1988) 389;

M. Aizawa, M. Matsuzawa and H. Shinohara, Thin Solid Films 160 (1988) 477.

[3] J.D. Swaten, Thin Solid Films 160 (1988) 197.

[4] Optical Linewidths in Glasses, J. Luminescence 36, No, 4,5 (1987).

[5] I. Zschokke, ed., Optical spectroscopy of glasses (Reidel, Dordrecht, 1986).

[6] R.M. MacFarlane and R.M. Shelby, J. Luminescence 36 (1987) 179

[7] R. Silbey and K. Kassner, J. Luminescence 36 (1987) 283.

[8] H.G. Braun, H. Fuchs and W. Schrepp, Thin Solid Films 159 (1988) 301;

M.R. Buhaenko, M.J. Grundy, R.M. Richardson and S.J. Roser, Thin Solid Films 159 (1988) 253;

V. Vogel and Ch. Wöll, Thin Solid Films 159 (1988) 429.

[9] D. Möbius and H. Kuhn, Israel J. Chem. 18 (1979) 375.

[10] U. Bogner, G. Röska and F. Graf, Thin Solid Films 99 (1983) 257.

[11] L. Kador, D. Haarer and R.I. Personov, J. Chem. Phys. 86 (1987) 5300

[12] V.M. Agranovich, V.K. Ivanov, R.I. Personov and N.V. Rasumova, Phys. Letters A 118 (1986) 239.

[13] A.I.M. Dicker, M. Noort, H.P.H. Thijssen, S. Völker and J.H. van der Waals, Chem. Phys. Letters 78 (1981) 212.

[14] R. van den Berg, H. van der Laan and S. Völker, Chem. Phys. Letters 142 (1987) 535.

[15] Th. Sesselmann, W. Richter, D. Haarer and H. Morawitz, Phys. Rev. B 36 (1987) 7601 .

[16] Th. Sesselmann, W. Richter and D. Haarer, J. Luminescence 36 (1987) 283.

[17] H. Kuhn, D. Möbius and H. Bücher, in: Physical methods of chemistry, Vol. 1, Part IIIB, eds. A. Weissberger and B. Rossiter (Wiley, New York, 1972).

[18] H. Hada, R. Hanawa, A. Haraguchi and Y. Yonezawa, J. Chem. Phys. 89 (1985) 560.

[19] H.P.H. Thijssen, A.I.M. Dicker and S. Volker, Chem. Phys. Letters 92 (1982) 7.

[20] M. Orrit, D. Möbius, U. Lehmann and H. Meyer, J. Chem. Phys. 85 (1986) 4966.

[21 ] A. Elschner and H. Bässler, Chem. Phys. 123 (1988) 305; Y. Kanematsu, R. Shiraishi, S. Saikan and T. Kushida, Chem. Phys. Letters 147 (1988) 53. 
[22] M. Berg, C.A. Walsh, L.R. Narasimhan, K.A. Littau and M.D. Fayer, Chem. Phys. Letters 139 (1987) 66.

[23] K.K. Rebane and A.A. Gorokhovskii, J. Luminescence 36 (1987) 237.

[24] H. Kuhn, J. Chem. Phys. 53 (1970) 101.
[25] A.P. Marchetti, M. Scozzafava and R.H. Young, Chem. Phys. Letters 51 (1977) 424.

[26] A. Renn, S.E. Bucher, A.J. Meixner, E. Meister and U. Wild, J. Luminescence 39 (1988) 181. 